\title{
Two-Photon-Absorption-Based OSNR Monitor for NRZ-PSK Transmission Systems
}

\author{
Douglas A. Reid, Krzysztof Bondarczuk, Karl J. Dexter, Student Member, IEEE, Kai Shi, \\ Prince M. Anandarajah, Member, IEEE, Liam P. Barry, Member, IEEE, Wei-Hua Guo, John O’Dowd, \\ Michael Lynch, A. Louise Bradley, and John F. Donegan, Member, IEEE
}

\begin{abstract}
A two-photon absorption microcavity-based technique for monitoring in-band optical signal-to-noise ratio (OSNR) in nonreturn-to-zero phase-shift-keying systems is presented. Experiments using a 10-Gb/s differential phase-shift-keying system showed that accurate measurements $( \pm 1 \mathrm{~dB})$ were possible for OSNRs in excess of $20 \mathrm{~dB}$.
\end{abstract}

Index Terms-Optical performance monitoring, optical signal-to-noise ratio (OSNR) measurement, phase-shift keying (PSK), two-photon absorption (TPA).

\section{INTRODUCTION}

$\mathbf{R}$ ECENTLY, phase-shift-keying (PSK) modulation formats have become popular in optical transmission systems because of their sensitivity improvement over standard on-off keying (OOK) and their potential for greater spectral efficiency [1]. In such systems, it remains important to monitor the optical signal-to-noise ratio (OSNR) throughout the network. It has been shown that the introduction of amplified spontaneous emission (ASE) noise near the start of a transmission link has a larger effect on PSK than equivalent OOK systems because it results in detrimental nonlinear phase noise as it copropagates with the signal [2]. OSNR is often measured spectrally by comparing the optical power within and between channels. This out-of-band measurement becomes difficult to use in high density wavelength-division-multiplexed (WDM) systems where the spectral efficiency is high, and for systems where optical filtering renders an in-band noise level which is significantly different from that found out-of-band. To overcome this, several techniques have been recently proposed

Manuscript received July 27, 2009; revised October 22, 2009; accepted December 02, 2009. First published January 12, 2010; current version published February 03,2010 . This work was conducted under the framework of the INSPIRE programme, funded by the Irish Government's Programme for Research in Third Level Institutions, Cycle 4, National Development Plan 2007-2013 and by the SFI funded CSET, Centre for Telecommunications Value Driven Research (CTVR).

D. A. Reid, K. Bondarczuk, K. J. Dexter, K. Shi, P. M. Anandarajah, and L. P. Barry are with the Research Institute for Networks and Communications Engineering, Dublin City University, Dublin, Ireland (e-mail: douglas.reid@dcu.ie; krzysztof@eeng.dcu.ie; karl.dexter2@mail.dcu.ie; kaishi@eeng.dcu.ie; anandara@eeng.dcu.ie; liam.barry@dcu.ie).

W.-H. Guo, J. O'Dowd, M. Lynch, A. L. Bradley, and J. F. Donegan are with the Semiconductor Photonics Group and CTVR, School of Physics, Trinity College Dublin, Dublin, Ireland (e-mail: guow@tcd.ie; jodowd@tcd.ie; lynchmi@tcd.ie; louise.bradley@tcd.ie; jdonegan@tcd.ie).

Color versions of one or more of the figures in this letter are available online at http://ieeexplore.ieee.org.

Digital Object Identifier 10.1109/LPT.2009.2038718 including the orthogonal delayed-homodyne method [3], the polarization nulling method [4], and polarization diversity technique [5]. These techniques show great promise, however, they do have some draw-backs. The orthogonal delayed-homodyne method requires a high-frequency receiver and radio-frequency (RF) spectrum analyzer, two relatively expensive components. In contrast, [4] and [5] have low component costs but rely on the assumption that the ASE is completely unpolarized; this assumption has been shown to generate significant errors in systems with polarized noise [6]. In this letter, we present a simple technique for monitoring OSNR in nonreturn-to-zero (NRZ)-PSK systems based on two-photon absorption (TPA) in a semiconductor microcavity.

\section{PRINCIPLE}

TPA is a nonlinear absorption process which occurs when incident photons have less energy than the semiconductor bandgap but greater than half the band-gap, $E_{g} / 2<h f<E_{g}$. This means that the absorption of two photons results in the generation of a single electron-hole pair. The resulting generated photocurrent is proportional to the incident electric field to the power of 4 . This means that the TPA photocurrent is a function of the peak power and duty cycle of the incident optical signal. This fact has been used to implement optical autocorrelators [7], and for the monitoring of dispersion [8]. More recently, it was shown that the TPA photocurrent is a function of the OSNR [9]. This can be understood if we consider the electric field of a quasi-monochromatic signal $E_{S}$ and that of spontaneous emission $E_{N}$. These can be represented as

$$
\begin{aligned}
E_{S}(t) & =\sqrt{2 A(t)} \cos \left(\omega_{0} t\right) \\
E_{N}(t) & =N_{A}(t) \cos \left(\omega_{0} t\right)-N_{B}(t) \sin \left(\omega_{0} t\right)
\end{aligned}
$$

where $N_{A}$ and $N_{B}$ are independent random variables with Gaussian probability density functions (zero mean and $\sigma^{2}$ variance). Because these electric fields have quite different probability density functions when we calculate their moments we find

$$
\begin{aligned}
\overline{\left\langle E_{S}^{2}\right\rangle} & =A \\
\overline{\left\langle E_{S}^{4}\right\rangle} & =\frac{3}{2} A^{2} \\
\overline{\left\langle E_{N}^{2}\right\rangle} & =\sigma^{2} \\
\overline{\left\langle E_{N}^{4}\right\rangle} & =3 \sigma^{4} .
\end{aligned}
$$




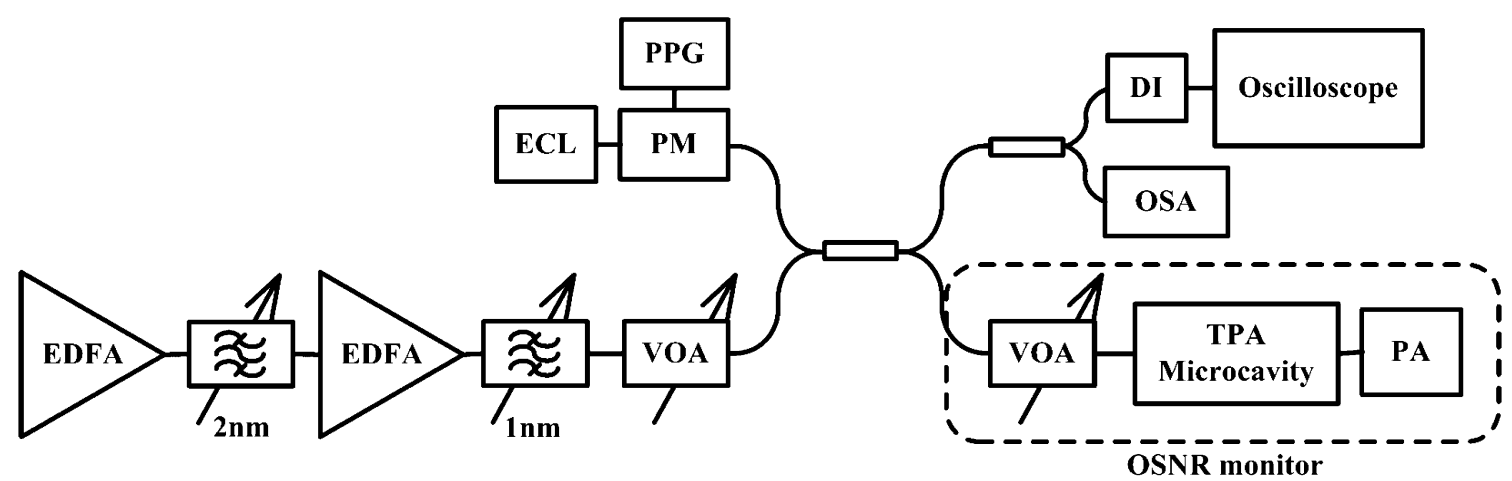

Fig. 1. Experimental setup for 10-Gb/s NRZ-DPSK OSNR monitor.

From these expressions and since

$$
\begin{array}{r}
P \propto \overline{\left\langle E^{2}\right\rangle} \\
i_{\mathrm{TPA}} \propto \overline{\left\langle E^{4}\right\rangle}
\end{array}
$$

where $P$ is the optical power and $i_{\mathrm{TPA}}$ is the TPA photocurrent it follows that

$$
\begin{aligned}
\left(i_{\mathrm{TPA}}\right)_{S} & \propto \frac{3}{2} P_{S}^{2} \\
\left(i_{\mathrm{TPA}}\right)_{N} & \propto 3 P_{N}^{2} .
\end{aligned}
$$

The result is that spontaneous emission generates twice the TPA photocurrent as a coherent continuous-wave (CW) signal of the same average power. This can be intuitively understood if you consider the higher peak-to-average-power ratio of a signal dominated by intensity noise when compared to a noise free signal. If both noise and a modulated signal are incident on the detector, the total time averaged photocurrent can be expressed as

$$
i_{p h}=C_{2} \bar{P}^{2}\left[1+\left(\frac{r}{1+r}\right)^{2}\left(\frac{1}{2 D}-1\right)\right]+C_{1} \bar{P}
$$

where $\bar{P}$ is the average optical power. $r$ is related to the OSNR (in decibels) by

$$
r=10^{\mathrm{OSNR} / 10}\left(0.1 \mathrm{~nm} / N_{\mathrm{BW}}\right)
$$

where $N_{\mathrm{BW}}$ is the noise equivalent bandwidth. $D$ is the signals effective duty cycle given by

$$
D=\overline{P(t)}^{2} / \overline{P(t)^{2}}
$$

and $C_{1}$ and $C_{2}$ are coefficients relating to the single and TPA efficiencies, respectively. This means that if the effective duty cycle is known, then the TPA photocurrent can be used to find the OSNR. In many cases, the exact duty cycle is not known so the TPA photocurrent cannot be used to uniquely define the OSNR. In addition, for the special case of NRZ-OOK, if $D=0.5$, then the TPA photocurrent is independent of the OSNR. However, in NRZ-PSK systems where no intensity modulation is present [for transmitters using phase modulators (PMs)], the duty cycle is known to be 1 and, therefore, $D$ can be eliminated from (11). For NRZ-PSK transmitters using Mach-Zehnder modulators, the short dips in intensity at the transitions results in a duty cycle less than one; however, knowledge of the RF amplifier and modulator rise-times can also be used to accurately calculate $D$ which can be used to solve (11). Because of the high speed of the TPA process, this means that the in-band ONSR can be monitored independently of the data rate. Rearranging (11) and setting $D$ equal to unity gives an expression for the OSNR

$$
r=\frac{\sqrt{2\left(1-\frac{i_{\mathrm{ph}}-C_{1} \bar{P}}{C_{2} \bar{P}^{2}}\right)}}{1-\sqrt{2\left(1-\frac{i_{\mathrm{ph}}-C_{1} \bar{P}}{C_{2} \bar{P}^{2}}\right)}} .
$$

Unfortunately, dispersion will cause a small reduction in $D$, resulting in an underestimate of the OSNR. However, for short reaches and longer dispersion-compensated systems, this effect is small. Assuming the dominant error is in the photocurrent measurement, the uncertainty in the measured OSNR value is given by

$$
\left(\sigma_{r}\right)_{\mathrm{dB}}=10 \log _{10}\left(\frac{\sigma_{i}}{C_{2} \bar{P}^{2}}\left(\frac{(1+r)^{3}}{r^{2}}\right)+1\right)
$$

where $\sigma_{i}$ is the variance in the photocurrent measurement.

\section{EXPERIMENT}

The setup shown in Fig. 1 was used to demonstrate the TPA OSNR monitoring technique experimentally. An external cavity laser (ECL) at $1558.7 \mathrm{~nm}$ was used in conjunction with a $\mathrm{LiNbO}_{3}$ optical PM and a 10-Gb/s $2^{7}-1$ PRBS data source (PPG) to form a 10-Gb/s NRZ differential phase-shift-keying (NRZ-DPSK) transmitter. A cascade of two erbium-doped fiber amplifiers (EDFAs) followed by a 1-nm filter and a variable optical attenuator (VOA) was then used to introduce a known amount of band-limited ASE to the signal, thereby degrading the OSNR. The resulting signal was passed to the OSNR monitor, and a DPSK receiver for eye measurements. An OSA was used for verification of the OSNR. The OSNR monitor was formed by a GaAs microcavity TPA photodetector [10] followed by a picoammeter (PA). A second VOA was used to ensure that optical power onto the TPA photodetector remained at a constant average power of 5-dBm independent of the OSNR. The polarization of the PSK signal was aligned 


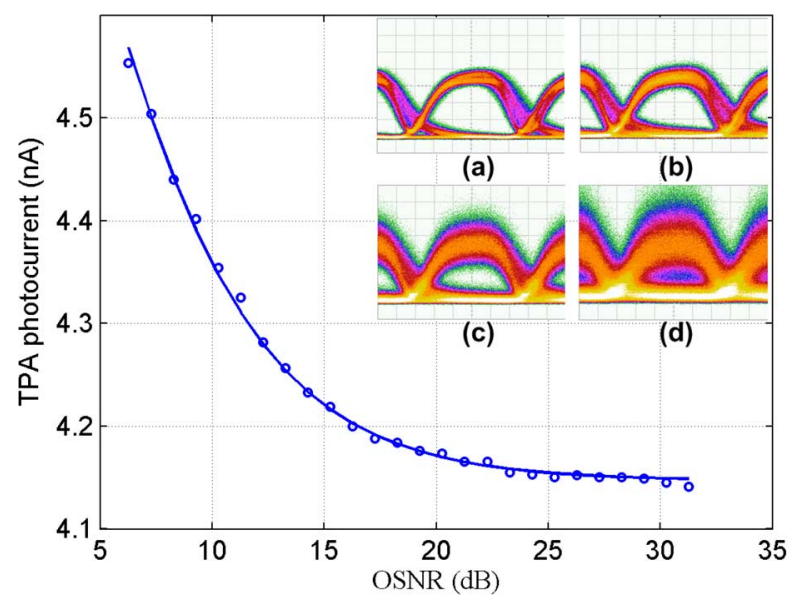

Fig. 2. Measured photocurrent from TPA microcavity photodiode as a function of signal OSNR (0.1-nm bandwidth), for a constant optical power of $5 \mathrm{dBm}$. Fitted curve using (11). Inset: Received eye diagrams of NRZ-DPSK signal for various in-band OSNRs: (a) $21.1 \mathrm{~dB}$; (b) $18.5 \mathrm{~dB}$; (c) $11 \mathrm{~dB}$; (d) $6.3 \mathrm{~dB}$.

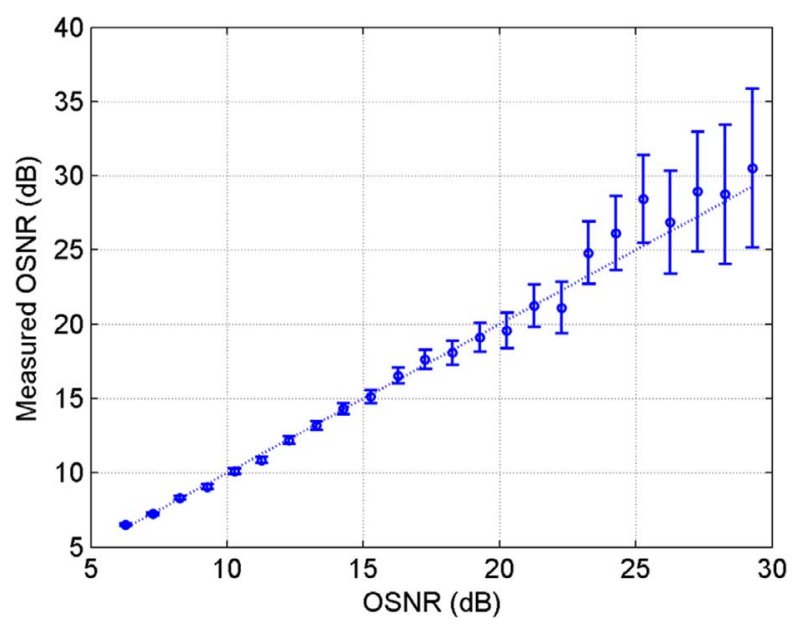

Fig. 3. Measured OSNR as a function of actual OSNR. Error bars calculated using equation ( 7 ) for \pm 10 -pA photocurrent uncertainty.

to maximize the TPA photocurrent, while the ASE was left unpolarized.

Fig. 2 shows the variation of the measured photocurrent as the OSNR was varied along with a fitted curve given by (11). Since ASE generates twice as much TPA photocurrent as a CW signal with the same average optical power, the total measured photocurrent is seen to increase by $500 \mathrm{pA}$ as the OSNR is decreased from 30 to $6 \mathrm{~dB}$ while the average optical power is kept constant. Calibration measurements gave the values for $C_{1}$ and $C_{2}$ of $9.26 \times 10^{-7} \mathrm{~A} / \mathrm{W}$ and $2.44 \times 10^{-4} \mathrm{~A} / \mathrm{W}^{2}$, respectively. The OSNR values calculated by (14) are shown in Fig. 3; the results have good agreement allowing $\pm 1-\mathrm{dB}$ measurements for OSNRs in excess of $20 \mathrm{~dB}$ for a photocurrent measurement with an error of $\pm 10 \mathrm{pA}$.

To highlight the effect of this OSNR reduction on system performance, Fig. 2 insets (a) and (b) show received eye-dia- grams following a DPSK demodulator [implemented using a 1-bit delay interferometer (DI)] for a selection of OSNRs. As expected, the eye is seen to close with the addition of ASE, which would result in a significant increase in bit-error rate. The TPA microcavity used had a resonance enhanced bandwidth of $1.2 \mathrm{~nm}$ centered at $1558.7 \mathrm{~nm}$ which can be tuned by $\sim 5 \mathrm{~nm}$ with adjustment of the incident optical angle. While this is nonoptimal for this application, the cavity mirrors could potentially be designed to accommodate a smaller channel bandwidth thereby increasing the enhancement of the TPA process. This would allow the monitoring of a single WDM channel. One drawback of TPA is its polarization dependence [11]; however, this could be overcome with a polarization diversity configuration.

\section{CONCLUSION}

We have presented a novel technique for the monitoring of OSNR in NRZ-PSK transmission systems using TPA in a semiconductor microcavity. Experiments using a 10-Gb/s DPSK system show that monitoring of OSNRs up to and above $20 \mathrm{~dB}$ would be possible with less then 1-dB error. The technique has the advantages of simplicity, low cost, and ease of integration and in principle is insensitive to data rate.

\section{REFERENCES}

[1] W. A. Atia and R. S. Bondurant, "Demonstration of return-to-zero signaling in both OOK and DPSK formats to improve receiver sensitivity in an optically preamplified receiver," in Proc. Lasers and Electro-Optics Society 12th Annual Meeting (LEOS '99), 1999, pp. 226-227.

[2] H. Kim and A. H. Gnauck, "Experimental investigation of the performance limitation of DPSK systems due to nonlinear phase noise," IEEE Photon. Technol. Lett., vol. 15, no. 2, pp. 320-322, Feb. 2003.

[3] C. J. Youn, K. J. Park, J. H. Lee, and Y. C. Chung, "OSNR monitoring technique based on orthogonal delayed-homodyne method," IEEE Photon. Technol. Lett., vol. 14, no. 10, pp. 1469-1471, Oct. 2002.

[4] J. H. Lee, D. K. Jung, C. H. Kim, and Y. C. Chung, "OSNR monitoring technique using polarization-nulling method," IEEE Photon. Technol. Lett., vol. 13, no. 1, pp. 88-90, Jan. 2001.

[5] T. B. Anderson, K. Clarke, S. D. Dods, and M. Bakaul, "Robust, low cost, in-band optical signal to noise monitoring using polarization diversity," in Proc. Optical Fiber Communication and the National Fiber Optic Engineers Conf., Mar. 2007, pp. 1-3.

[6] M. D. Feuer, "Measurement of OSNR in the presence of partially polarized ASE," IEEE Photon. Technol. Lett., vol. 17, no. 2, pp. 435-437, Feb. 2005.

[7] Y. Takagi, T. Kobayashi, K. Yoshihara, and S. Imamura, "Multiple- and single-shot autocorrelator based on two-photon conductivity in semiconductors," Opt. Lett., vol. 17, no. 9, pp. 658-660, 1992.

[8] S. Wielandy, M. Fishteyn, and B. Zhu, "Optical performance monitoring using nonlinear detection," J. Lightw. Technol., vol. 22, no. 3, pp. 784-793, Mar. 2004.

[9] W. H. Guo, J. O’Dowd, M. Lynch, A. L. Bradley, J. F. Donegan, L. P. Barry, and D. C. Kilper, "Two-photon absorption generated by optically amplified signals," Electron. Lett., vol. 44, no. 18, pp. 1087-1088, Aug. 2008.

[10] H. Folliot, M. Lynch, L. P. Barry, A. L. Bradley, L. A. Dunbar, J. Hegarty, J. F. Donegan, J. S. Roberts, and G. Hill, “Two-photon absorption photocurrent enhancement in bulk AlGaAs semiconductor microcavities," Appl. Phys. Lett., vol. 80, pp. 1328-1330, 2002.

[11] J. O' Dowd, W. H. Guo, E. Flood, M. Lynch, A. L. Bradley, L. P. Barry, and J. F. Donegan, "Polarization dependence of a GaAs-based two-photon absorption microcavity photodetector," Opt. Express, vol. 16, pp. 17682-17688, 2008. 